\title{
Narrow-Line Regions in Seyfert Galaxies and Quasars: Modelling 2D Kinematics
}

\section{Bruno Jungwiert ${ }^{1,2}$, Ivana Stoklasová ${ }^{1,3,4}$, and Nicola Bennert ${ }^{2,5}$}

${ }^{1}$ Astronomical Institute, Academy of Sciences of the Czech Republic, Prague, Czech Republic

${ }^{2}$ Institute of Geophysics and Planetary Physics, University of California-Riverside, USA

${ }^{3}$ Faculty of Mathematics and Physics, Charles University in Prague, Czech Republic

${ }^{4}$ Centre de Recherche Astronomique de Lyon-Observatoire de Lyon, Université Lyon 1, France

${ }^{5}$ Department of Physics and Astronomy, University of California-Riverside, USA

\begin{abstract}
We attempt to provide new insights into the kinematics of narrow-line regions (NLR) in Seyfert galaxies and quasars, the understanding of which, as well as of their intrinsic 3D morphology, is still rather limited. Motivated by the rise of the integral-field spectroscopy, capable of mapping full 2D velocity fields, and by our observational projects on NLRs (see e.g. Stoklasová et al., this volume) we have developed a computer code producing 2D kinematical maps resulting from projections of various gas geometries and velocity fields.
\end{abstract}

Keywords. galaxies: active, galaxies: Seyfert, galaxies: nuclei, galaxies: structure, galaxies: kinematics and dynamics, quasar: emission lines

Simulated geometries of gas include spheres, disks and rings/tori with various radial/vertical density profiles. Multi-component systems composed of an arbitrary combination of the above distributions, possibly misaligned with each other, are allowed. The modelled line-of-sight velocity distributions reflect rotation/outflows/random motions. The rotation curve is due to several components: super-massive black hole, stellar bulge/disk and dark matter halo. An ionization bi-cone (parametrized by opening angle $\theta$, two orientation angles and length) can be superposed: the emission is then assumed to originate only within the bi-cone. Dust obscuration can be taken into account.
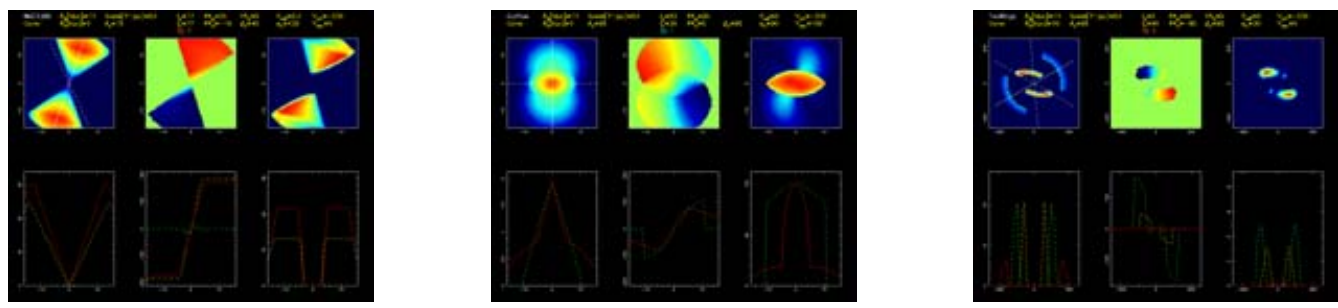

Figure 1. Examples of 3 NLR models (sub-panels show 2D maps and 1D profiles of emission-line intensity, mean LOS velocity and velocity dispersion). Left: differentially rotating disk $\left(I_{\text {disk }}=77^{\circ}, P A_{\text {disk }}=25^{\circ}\right)+$ ion. cone $\left(\theta=70^{\circ}, I_{\text {cone }}=77^{\circ}, P A_{\text {cone }}=-19^{\circ}\right)$ (the galaxy is observed as Sy 2); Middle: sphere + ion. cone $\left(\theta=90^{\circ}, I_{\text {cone }}=60^{\circ}\right)+$ outflow along the cone (observed as Sy 1); Right: two misaligned rotating rings + ion. cone (observed as Sy 2).

\section{Acknowledgements}

This project has been supported by the Institutional Research Plan No. AV0Z10030501 of the Academy of Sciences of the Czech Republic. B.J. was also supported by the EU Research Training Network "Euro3D" (HPRN-CT-2002-00305). I.S. gratefully acknowledges the PhD Fellowship of the French Government. 